\title{
FACTORS AFFECTING SURVIVAL OF RHIZOBIA ON INOCULATED CLOVER SEED
}

\author{
G. G. Taylor and J. M. Lloyd \\ Fruitgrowers Chemical Co. Ltd., Mapua, Nelson
}

INTRODUCTION

Production of rhizobium inoculated and coated lucerne and clover seed was commenced in 1965 by Coated Seed $\mathrm{Ltd}$. in response to a suggestion from the Department of Scientific and Industrial Research that it would be a profitable undertaking and would serve the needs of the farming community. Events proved that neither objective could be easily achieved and it became necessary to investigate the seed-inoculum-coat complex in relation to longevity of the rhizobia and to devise manufacturing procedures which would not only meet the objective of effective nodulation in the field but would be economic and provide an acceptable commercial product. In the course of this work on behalf of Coated Seed Ltd., certain principles have been defined which are of interest to research workers and farmers.

Successful nodulation of clover seedlings has not always followed the use of inoculated seed. Perhaps use of the word "inoculated" has led to misunderstanding because mere treatment of seed with a culture of rhizobia is not enough to ensure infection. Infection is something which happens later, after seed has germinated and the bacteria have entered the root hairs and become established within the plant tissue. It is apparent that, unless viable rhizobia in sufficient numbers remain on the seed at time of planting, there is little chance of successful nodulation other than by rhizobia al ready in the soil. Between inoculation and seedling establishment many factors leading to death of rhizobia can intervene. Attempts to define causes of impaired viability of inoculum up to time of planting have been made and results are reported here with specific reference to white clover.

\section{MATERIALS AND METHODS}

In most of this work, commercial "Adlife" inoculum was used. More recently strains of Rhizobium trifolii have been obtained direct from the Plant Diseases Division, 
D.S.I.R., Auckland. Within the species Rhizobium trifolit several different strains are recognized. The strain N.Z.29, first produced at the Plant Diseases Division, has been used successfully in New Zealand and overseas for many years. Following work in Australia, the Plant Diseases Division has confirmed that the strain T.A.I. is a better competitor in the field and is more reliable in culture and this strain has now been adopted for use in N ew Zealand.

In the course of culturing rhizobia, changes can take place which, though not showing any visual differences, may lead to failure in nodulation or, even though nodulation succeeds, to loss of effective nitrogen fixation. To guard against this, a series of mother cultures, prepared from original isolations from growing plants, are used to provide for bulk production of inoculum over several months. During this time, inoculated plants are grown under sterile conditions and the strain re-isolated from large nodules from vigorously growing plants.

Numbers of bacteria at time of seed inoculation have not always been determined, but in all trials loadings of bacteria between different treatments have been kept constant. Where numbers of bacteria in cultures are re ported, these were measured by normal methods of serial dilution of inoculum and counting colonies developed on agar plates,

Success or failure of treated seed to produce nodules on seedlings has been measured by growing individual seeds on sterilized nitrogen-free nutrient agar in testtubes. This method has been preferred to those of estimating numbers of bacteria since it reveals the nodulating potential of the seed-inoculum-coating complex as a whole. Normally 30 seeds have been used for each treatment and recorded as nil or nodulated, irrespective of the numbers of nodules formed.

Gafsa phosphate, dolomite and lime $\left(+98 \% \mathrm{CaCO}_{3}\right)$ coating materials were milled to a particle size of plus $95 \%$ passing a 300 mesh B.S. sieve.

\section{SURVIVAL OF RHIZOBIA ON SEED}

Immediately following treatment of seed, death of rhizobia commences. The rate at which this proceeds de pends on a series of inter-related factors.

TEMPERATURE

A practical necessity in inoculating and coating is that seed be dried and, since heat is necessary, it is important 
that this should not be harmful to the rhizobia. Suspensions of Rhizobium trifolii when held for 10 minutes at different temperatures have been found to survive 43.X. Tests have shown that inoculated seed can be held for 12 hours at $32^{\circ} \mathrm{C}$ without measurably affecting nodulation capacity and this is taken as a maximum for seed in course of drying.

\section{MOISTURE}

In the production process, drying of the coat is necessary, and to determine the effect on survival of rhizobia a series of samples of coated seed were dried to different levels of total water content and measured for nodulating capacity. The samples were held in sealed bottles and tested over a period of 120 days. Results given in Fig. 1 show that survival of rhizobia increased as moisture was reduced. Similar results have frequently been obtained

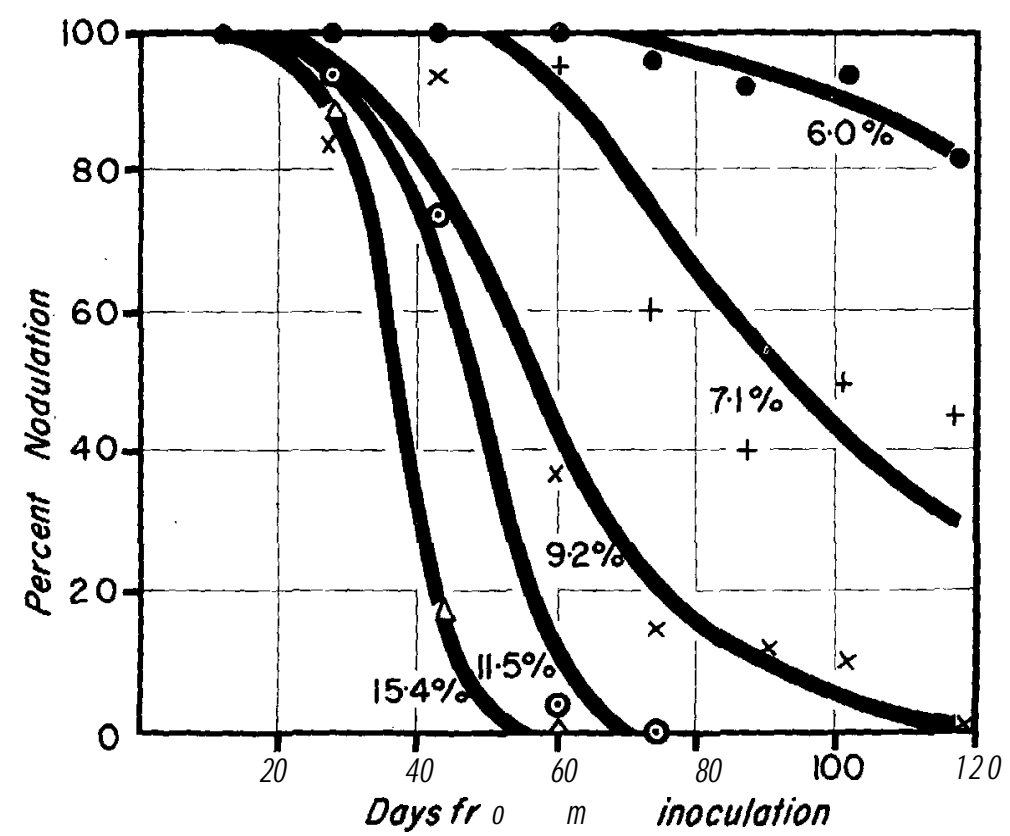

FIG, 1: Nodulating capacity of inoculated white clover seed coated with Gafsa-dolomite and dritd to different levels of total moisture shown in per cent. 


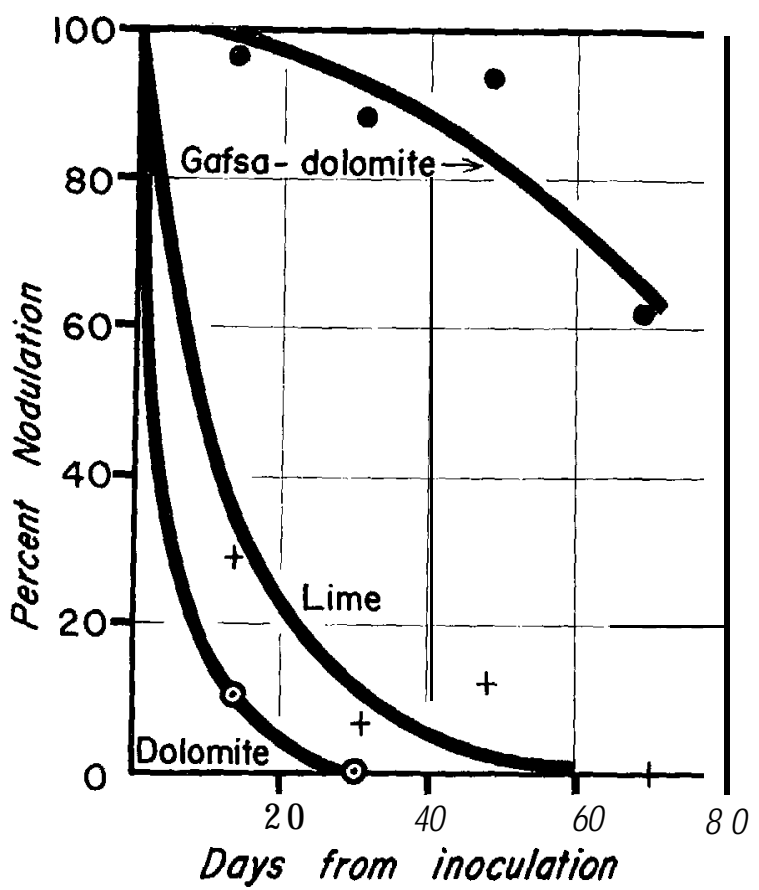

FiG. 2: Nodulating capacity of inoculated white clover seed when coated with Gafsa-dolomite, lime and dolomite.

when checking production samples before and after drying. Where seed is inoculated but not coated, rhizobia may survive in the dried seed for up to 14 days to give partial nodulation yet die within one or two days on the wet seed.

\section{Coating Materials}

Various adhesives have been tested for use in attaching coating materials to the seed. A wide range of materials is available for this purpose - e.g., methyl cellulose, gum arabic, sugars. A nimal glues can also be used but where they contain preservatives are very harmful to rhizobia.

Results of a trial with Gafsa phosphate plus dolomite, lime alone, and dolomite alone as coating materials are shown in Fig. 2. The improved persistence of nodulating capacity from the Gafsa-dolomite mixture in this and other trials has led to the abandonment of lime as a coating material on clover seed. This does not apply to lucerne seed where lime is still used. 
Use of a peat-lime mixture as a coating material led to an immediate improvement in rhizobia viability. Subsequently Gafsa-dolomite was used in place of lime and a process developed whereby the peat was interposed be tween the inoculum on the seed and the outer coat of Gafsa-dolomite. Results illustrated in Fig. 3 show clearly the improved nodulating capacity of seed where the peat layer was used as compared with Gafsa-dolomite alone. Where seed was inoculated but not coated, rhizobia were short-lived.

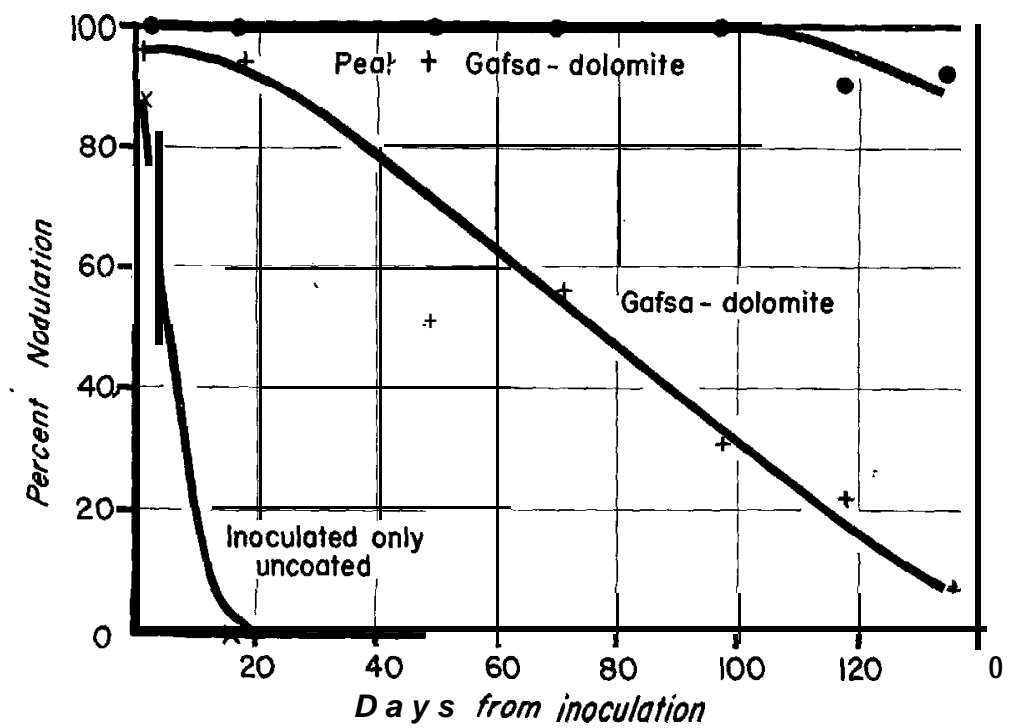

FIG. 3: Nodulating capacity of inoculated white clover seed comparing uncoated, Gafsa-dolomite and peat Gafsa-dolomite coated.

\section{EXPOSURE TO SUNLIGHT}

A trial to measure the likelihood of sunlight affecting rhizobia was made on inoculated seed coated with Gafsadolomite and with peat plus Gafsa-dolomite. The seed was set out on soil in petri dishes in direct sunlight and checked for nodulating capacity at various intervals with results as shown in Table 1. It is apparent that rhizobia are severely affected by sunlight and that use of peat delays but does not prevent this effect. 
TABLE 1: EFFECT OF SUNLIGHT ON NODULATING CAPACITY OF INOCULATED WHITE CLOVER SEED COATED WITH GAFSADOLOMITE AND WITH PEAT PLUS GAFSA-DOLOMITE.

\begin{tabular}{ccc}
\hline Exposure to Bright & \multicolumn{3}{c}{$\%$ N odulation } \\
Sunlight $(h r)$ & Gafsa-dolomite & Feat + Gajsa-dolomite \\
\hline 5 & 15 & 100 \\
10 & 16 & 96 \\
15 & 10 & 76 \\
25 & nil & 44 \\
\hline
\end{tabular}

Exposure to Superphoshrate

Samples of bare inoculated seed, inoculated plus Gafsadolomite, and inoculated plus peat plus Gafsa-dolomite were prepared and dried and were then mixed with superphosphate at the rate of 1 part treated seed to 10 parts of superphosphate. The phosphate had a moisture content of $5.9 \%$ and only that fraction passing a 22 mesh B.S. sieve was used. Samples of the mixture were removed at different intervals of time, vigorously screened to separate seed and super-phosphate and the seed checked for nodulation. Results given in Table 2 show that rhizobia on bare inoculated seed are quickly killed, that Gafsadolomite provides some protection, and the use of peat in the coat substantially reduces damage from superphosphate.

TABLE 2: EFFECT OF SUPERPHOSPHATE ON NODULATING CAPACITY OF INOCULATED WHITE CLOVER SEED UNCOATED, WITH GAFSA-DOLOMITE AND WITH PEAT PLUS GAFSADOLOMITE.

\begin{tabular}{|c|c|c|c|}
\hline $\begin{array}{l}\text { Exposure Time to } \\
\text { Superphosphate (hr) }\end{array}$ & Uncoated & Gafsa-dolomite & $\begin{array}{c}\text { Peat plus } \\
\text { Ga/sa-dolomite }\end{array}$ \\
\hline 1 & $\begin{array}{ll}- & \text { nil }\end{array}$ & $3 \overline{4}$ & 100 \\
\hline 2 & nil & 10 & 100 \\
\hline 4 & nil & 9 & 91 \\
\hline 8 & nil & 3 & 84 \\
\hline 24 & nil & nil & 63 \\
\hline
\end{tabular}

\section{EFFECT OF TYPE OF INOCULUM}

Results of a trial in which inoculum loading was varied from approximately five times the normal commercial rate down to 0.01 times this rate are shown in Fig. 4. 


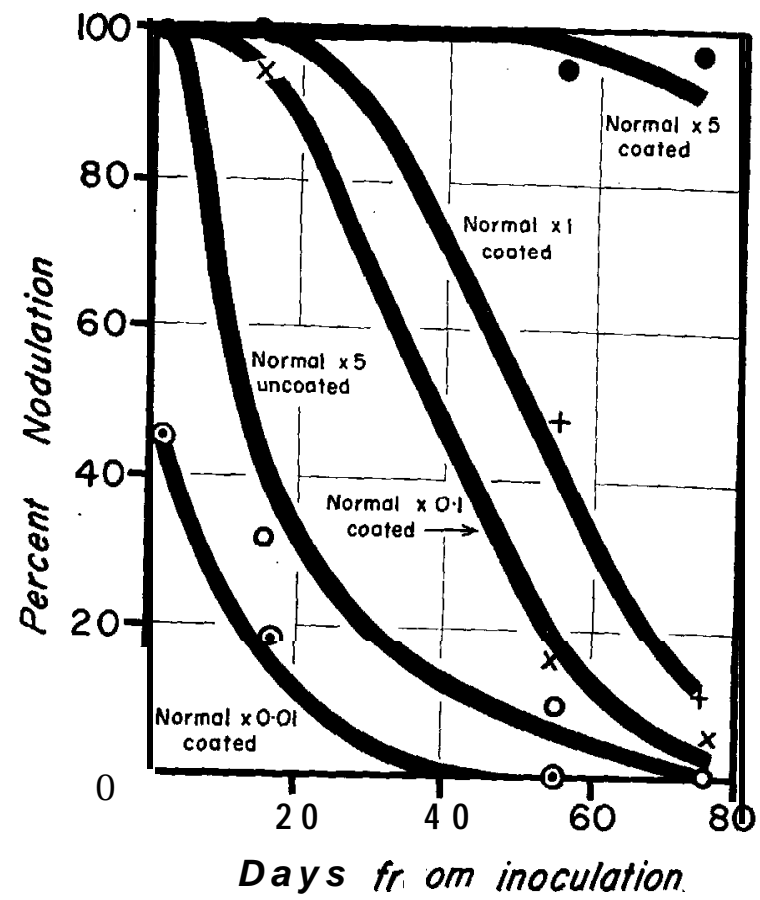

FIG. 4: Nodulating capacity of white clover seed inoculated at $\times 5$ normal rate down to $\times 0.02$ normal rate and coated with peat plus Galsadolomite.

Sharp differences in survival of nodulating capacity are shown but, even at five times normal commercial rate of inoculum, nodulation failed sooner than is usually found. This suggested that methods of growing Rhizobium trifolii mght have some influence on survival on coated seeds.

Cultures of rhizobium for inoculation purposes can be produced on a variety of media, typical of which are nutrient agar, liquid broth, in broth subsequently added to peat, and directly in nutrient peat. On nutrient agar, rate of multiplication of rhizobia is rapid but is followed by a rapid decline. On peat, multiplication is slower but rate of decline is also slow, with the net result that cultures have been found to increase in numbers when held in cool store $\left(4.5^{\prime \prime}\right.$ to $\left.7^{\circ} \mathrm{C}\right)$ over a period of four months to levels far in excess of those on agar.

In order to test longevity on inoculated seed, numbers of bacteria in three types of inoculum, namely, nutrient agar, broth culture in peat and nutrient peat, were counted. Seed was then inoculated at a rate of approximately 
RHIZOBIA ON INOCULATED CLOVER SEED

3,000 bacteria per seed and coated with peat plus Gafsadolomite. Nodulation counts shown in Fig. 5 indicate a substantial advantage in favour of the nutrient peat inoculum.

\section{RHIZOBIUM LOADINGS}

To ensure good nodule establishment of a selected strain of rhizobia, it is not sufficient that seed carry viable bacteria at time of sowing. There still remains an important period before seed has germinated and the selected strain established in nodules. Climatic conditions and the need to compete for nodulation sites against inefficient rhizobia present in many soils require loadings of bacteria in excess of those necessary for nodulation under laboratory conditions.

The question of what is a sufficiently heavy loading cannot be determined accurately because of the variable field conditions encountered. Evidence suggests, however, that the present minimum of approximately 250 bacteria per white clover seed as required for certification by the Plant Diseases Division, D.S.I.R., is too low for many situations.

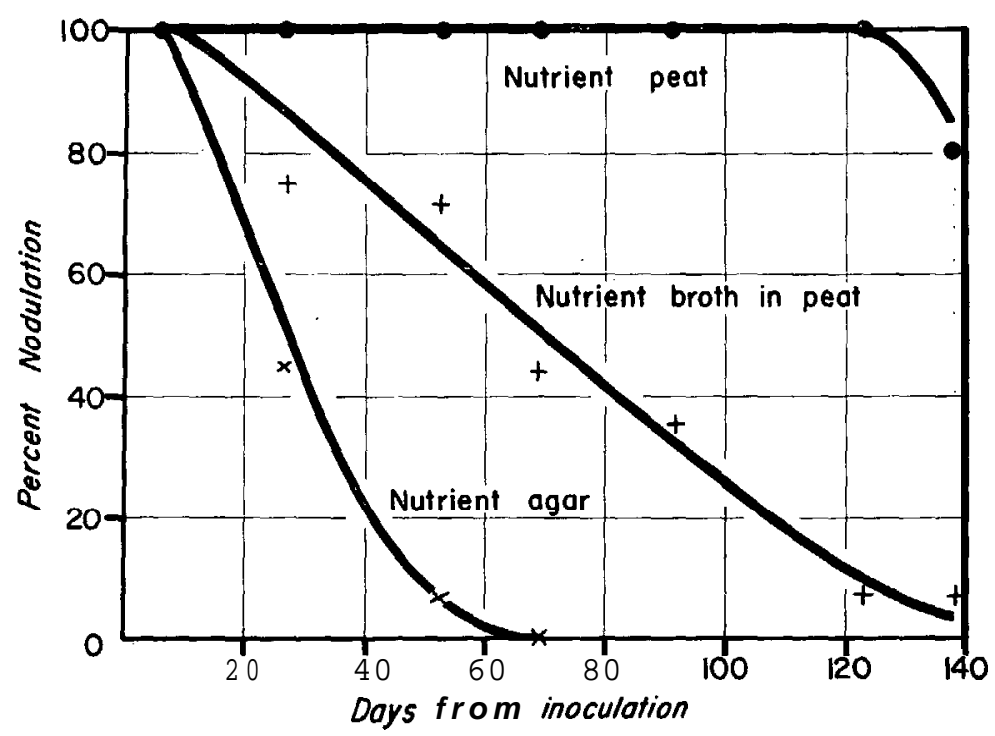

FIG. 5: Nodulating capacity of white clover seed inoculated with rhizobia grown on nutrient agar, in broth in peat, and in nutrient peat and coated with peat plus Gafsa-dolomite. 
In practice, commercial loadings at time of seed inoculation have usually provided for 1,500. to 3,000 bacteria per white clover seed. Whilst these rates of inoculation for coated seed may be sufficient for some situations.. even higher loadings seem desirable to provide sufficient numbers of bacteria in adverse conditions. To this end, investigations have been made into levels and types of nutrients in peat and results have shown that some substantial improvements can be made. This work is still in progress but it has already been possible to raise the loadIngs in commercial production to give at least 12,000 bacteria per white clover seed at time of treatment. Bacterial loadings of 30,000 per seed seem to be a reasonable objective.

\section{CONCLUSION}

In developing a process of inoculating and coating clover seed, the objective is to provide the maximum loading of viable rhizobia at the time of sowing. A number of factors have been shown to affect viability of the bacteria and many of these are inter-related. Improvement in one direction necessitates repetition of earlier work'in the light of new situations.

While the precise significance of different conditions cannot be stated, the general principles which have been applied lead to production of coated inoculated seed of much higher potential nodulating capacity than has previously been available. Some of the important factors which are recognized at present but may later be further defined, appear to be:

(1) Use of the best strain of Rhizobium trifolii grown in nutrient peat.

(2) Inoculation of seed at the heaviest loadings economically practicable.

(3) Application of peat in a protective layer.

(4) Application of Gafsa phosphate-dolomite as a final coat.

(5) Use of suitable adhesives which are not toxic to rhizobia.

(6) Drying at temperatures not harmful to rhizobia.

(7) Use of storage and transport facilities where temperatures are as low as practicable. 
(8) If coated seed is to be applied with superphosphate, sowing as soon after mixing as possible.

(9) Selection of conditions for over-sowing where some protection from direct sunlight can be obtained e.g., in low bracken rather than close burn-off.

(10) Sowing inoculated coated seed within two months of production.

\section{ACKNOWLEDGEMENTS}

Assistance and advice have been given by staff of the Department of Agriculture, Lincoln College, and the Department of Scientific and Industrial Research. The help of Dr D. Dye and A. Hastings in providing cultures and advising on techniques is gratefully acknowledged.

\section{DISCUSSION}

To the comment that results in Australia showed advantages in limepelleting white clover seed in contrast to the results obtained here, Taylor stated that perhaps the Australian results would have been better still had Gafsa-dolomite been used. It was suggested that out-of-date inoculum should be withdrawn from the market. Taylor said that his firm did not produce inoculum for commercial use but agreed that age was very important. In the trials described, the inoculum was never more than a fortnight old and so age and numbers of rhizobia were under complete control.

The inoculum used was from a commercial source and was essentially derived from N.Z. 29 strain. He agreed with a suggestion that more work should be done at other and lower $\mathrm{pH}$ values.

Questioned whether any work had been done on pea inoculum, Taylor stated that a small amount had been carried out and responses obtained under glasshouse conditions. He thought it would be uneconomic at present to produce a suitable inoculum for peas. A further complicating factor was the necessity to treat seed with fungicides which could affect the inoculum.

Asked whether standard rates of inoculum had been used in his studies, Taylor replied that much lower $(1 / 4$ normal $)$ rates had been applied to ensure attaining differences between coatings.

$\mathrm{He}$ had stated that coating improved the moisture status because it acted as an absorbent matrix which could possibly attract moisture to the seed. Admittedly, this had not occurred in some field trials, but he felt there were situations where it could be an advantage. 